\title{
Protée
}

\section{Interview avec Raoul Coutard}

\section{Pierre-Emmanuel Parais et Marie Renoue}

Volume 31, numéro 3, hiver 2003

\section{Lumières}

URI : https://id.erudit.org/iderudit/008441ar

DOI : https://doi.org/10.7202/008441ar

Aller au sommaire du numéro

Éditeur(s)

Département des arts et lettres - Université du Québec à Chicoutimi

\section{ISSN}

0300-3523 (imprimé)

1708-2307 (numérique)

Découvrir la revue

\section{Citer cet article}

Parais, P.-E. \& Renoue, M. (2003). Interview avec Raoul Coutard. Protée, 31(3), 101-106. https://doi.org/10.7202/008441ar

\section{Résumé de l'article}

Célèbre chef-opérateur de la " nouvelle vague ", photographe et réalisateur Raoul Coutard évoque, au milieu d'anecdotes sur des « films-cultes » du cinéma - Le Mépris, À bout de souffle,Alphaville ou Tirez sur le pianiste -, son travail d'éclairagiste, en particulier les contraintes techniques, chimiques rencontrées lors des tournages. Une discussion fort technique qui indique, au-delà des manques du langage de la lumière patents lors des tournages, combien les contraintes matérielles sont génératrices d'effets lumineux signifiants et admirables pour leurs spectateurs. d'utilisation que vous pouvez consulter en ligne.

https://apropos.erudit.org/fr/usagers/politique-dutilisation/ 


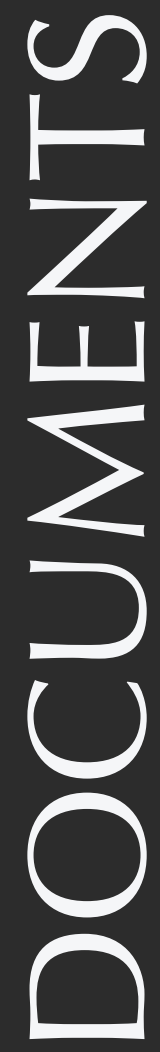

D'abord photographe des armées en Indochine, expérience qui transparaîtra dans son premier long-métrage comme cinéaste, Hoa Binh (1969), Raoul Coutard, né en 1924, est considéré comme le chef-opérateur emblématique de la «nouvelle vague ». Après trois films avec Pierre Schoendoerffer, il travaille sur À bout de souffle de Jean-Luc Godard, tourné en extérieurs dans les conditions du reportage, avec la caméra à l'épaule. Le tandem Godard-Coutard va rénover à lui seul l'esthétique du cinéma français, alors enfermé dans la lumière tamisée et conventionnelle du studio. Les teintes vivaces du Mépris (1963), le gris «eisenteinien » des Carabiniers (1963), les couleurs enchanteresses de Pierrot le fou (1965), les halos lumineux et le gris métallique d'Alphaville (1965), l'esthétique « pop art » de La Chinoise (1967) portent la marque de fabrique de Raoul Coutard. De 1960 à 1968, il tournera quatorze films avec Jean-Luc Godard, avant que leur association ne reprenne pour deux longsmétrages (Passion et Prénom Carmen) au début des années 1980. Sollicité par de nombreux cinéastes de la «nouvelle vague », Raoul Coutard a notamment travaillé avec François Truffaut (Tirez sur le pianiste, Jules et Jim, La Peau douce, La mariée était en noir) et Jacques Demy (Lola). Entre autres nombreuses distinctions, il a été désigné en 1988 meilleur directeur de la photographie des trente dernières années au sein de la communauté européenne. 


\section{INTERVIEW AVEC RAOUL COUTARD}

par Pierre-emmanuel Parais

ET MARIE Renoue

Que retenez-vous de votre expérience de chef-opérateur? Quels conseils pourriez-vous donner à celui qui désirerait affiner sa perception ou sa capture de la lumière?

Cela dépend du genre de film sur lequel on travaille, aussi de sa forme. Il est évident, par exemple, qu'on n'éclaire pas de la même façon un film en couleurs et un film en noir et blanc; étant donné l'absence de couleur, il faut qu'on transcende l'image d'une autre manière. Regardez les reportages de guerre, ceux de 1939-1945 et de I'Indochine, reportages qui tous ont été faits en noir et blanc. Je suis toujours sidéré par la qualité de leur photographie, d'autant que ces documents ont été pris en général sous le feu. Quand on fait ce genre de reportage, on se dit: "Tiens, je vais aller là parce que la lumière est bonne, s'il se passe quelque chose ce sera bien ». C'est-à-dire qu'il y a une tentative de donner une véracité avec une qualité de l'image. Quand vous regardez des documentaires d'aujourd'hui, tournés en couleurs, il y a ce qui est le contenu de l'image, le document. Point.

La recherche sur la lumière, au cinéma et plus largement dans l'audiovisuel, vous semble-t-elle pas suffisamment développée ou exploitée? Sinon, quelles auraient pu être ces recherches?

Dans tous les films que j'ai éclairés, je pense que les possibilités de l'image n'ont jamais été pleinement exploitées. Mais encore aurait-il fallu que l'on se penche sur la question. Car, finalement, les expérimentations faites sur l'image se sont restreintes à des modes. Il y a eu le flashage: à un moment donné, tous les films étaient flashés, avec des images un peu gris métallique. Maintenant vient la mode des bleus. Cela tient davantage d'une absence d'idées. Sur certains films en tout cas, en prenant le temps de régler les problèmes, on aurait pu tenter des effets que le numérique aujourd'hui permet de concevoir. Certains films français ou étrangers ont accordé une grande importance à l'image, mais je ne crois pas que ce soit finalement très important: il faudrait une plus grande sensibilité de chacun à l'image, mais comme le travail de la lumière n'est pas bien appréhendé par le public, l'amélioration de certains effets n'aurait pas été visible par tous. Et comme ces effets supposent une dépense, non seulement intellectuelle mais financière, on ne peut pas tirer toute la couverture à soi pour un film qui sera le résultat d'une collaboration entre équipes... Quant aux effets, je pense qu'il aurait été possible non pas de changer des couleurs, mais d'installer d'autres ambiances. Certes, il y a eu des tentatives dans ce sens: dans les films 
américains et certains films français (je pense à un de mes collègues qui faisait une très belle photographie, Bruno Nuytten), il y a eu une grande mode, celle d'éclairer, dans un appartement, les pièces voisines d'une autre couleur que la pièce où se jouait la scène, soit jaune, rougeâtre ou verdâtre. Mais la pièce de l'action était éclairée normalement. Il est vrai que jouer sur l'image entraîne des incidences sur les costumes et le décor, car, en argentique, les possibilités ne sont pas très étendues. En numérique, cela pourra se faire plus facilement.

Quel regard portez-vous, en l'occurrence, sur l'avènement du numérique?

Je trouve ça très bien. Le numérique offre des possibilités considérables. Ça permet d'effectuer des rectifications qui n'étaient pas envisageables en argentique, d'ajouter un peu de qualité à l'image. Le seul problème est que tout le monde va intervenir, comme dans la publicité. Les opérateurs français sont toujours en retard dans ce domaine: le confort des cartes professionnelles du Centre du cinéma les a dispensés de réfléchir à l'évolution de la profession. Mais maintenant, en post-production par exemple, quand quelqu'un d'autre va retoucher la lumière, qui sera le responsable de l'image?

L'image dont vous êtes responsable, celle qui est attachée à votre nom, est celle des films de la «nouvelle vague». Envisagiez-vous, dès vos premiers films avec Schoendoerffer, de faire une lumière autre que celle du cinéma français de studio?

Non, pas du tout. Quand Schoendoerffer m'a demandé de travailler sur La Passe du diable, je n'avais pas la moindre idée du métier de chef-opérateur. Si j'avais su d'ailleurs, je me serais dérobé! Mais ce premier film s'est avéré extrêmement simple, bien que, fait rarissime à l'époque, on l'ait tourné en cinémascope et en couleurs. Les problèmes véritables surviennent quand il s'agit de manipuler la lumière, mais là une maîtrise technique de la prise de vues photographiques ordinaire suffisait.

C'est donc avec À bout de souffle que votre approche de l'éclairage s'est affinée? La qualité de la photographie, des contre-jours en particulier, est souvent présentée comme remarquable.

Les données de départ $d^{\prime}$ À bout de souffle étaient de travailler sur le mode du reportage. En d'autres termes: caméra à l'épaule, pas de projecteur sur pied, et les ciné-flashs n'existaient pas à l'époque. II n'y a pas du tout de lumière dans le film. La qualité de l'image tient à la pellicule utilisée qui permet d'avoir une transparence dans les noirs; c'était de la pellicule classique, de la double X Kodak, la même que maintenant: sur le dernier film que j'ai tourné avec Garrel, la pellicule n’a pas changé. Pour les séquences de nuit, nous avons utilisé une pellicule anglaise très sensible, I'Ilford HPS, et nous l'avons développée dans un bain spécial à base de paraphénylènediamine, un révélateur qu'emploient les photographes pour augmenter la sensibilité, mais qui a la qualité de ne pas trop faire monter le grain.

Pour Alphaville, l'idée était manifestement de créer une lumière, un univers énergétique et artificiel. La pellicule était exposée "frontalement» aux sources lumineuses, aux enseignes, ampoules, phares de voiture ou à la seule flamme d'un briquet.

C'est effectivement un univers assez étrange, en noir et blanc. Nous avons utilisé comme pellicule la HPS. À l'époque d'À bout de souffle, elle n'existait que pour la photographie, ce qui nous a obligés à acheter des bobines de $20 \mathrm{~m}, 60 \mathrm{~m}, 10 \mathrm{~m}$. L'inconvénient portant sur la durée: avec $10 \mathrm{~m}$, on ne pouvait tourner que vingt secondes théoriquement, donc un plan de quinze secondes. Mais c'était le jeu. Pour Alphaville, par contre, la pellicule était disponible en cinéma. Au demeurant, la majorité des plans a été tournée avec une Mitchell, qui nécessitait une pellicule avec perforations cinéma. Tandis que le tournage avec un Cameflex, qui accepte n'importe quelles perforations, nous avait permis, dans À bout de souffle, d'utiliser la HPS photo. 
Comment aviez-vous convenu, avec Godard, des éclairages d'Alphaville, qui est un film sur la lumière, l'énergie? Avec Jean-Luc, les discussions ont lieu au début, des discussions rapides, une heure tout au plus. Ses films se fondent sur l'idée d'un challenge. Pour Les Carabiniers par exemple, il voulait que le film ressemble à une œuvre de cinémathèque. Donc on l'a développé avec un gamma plus élevé, avant de le tirer sur la pellicule-titre de façon à obtenir des blancs et des noirs avec très peu de gris. Comme s'il s'agissait d'un film tiré de nombreuses fois. Dans Alphaville, ce qui intéressait Jean-Luc était qu'on fasse le film en utilisant le minimum de lumière. C'est d'ailleurs son gros problème: essayer de travailler en employant presque toujours le moins de lumière possible, le moins de sources, qu'on ait tendance à moins bricoler la lumière. Dans Alphaville, le plus gros problème a été d'ordre complètement technique: I'absence d'anti-halo sur les pellicules noir et blanc. Sur les pellicules couleurs, indépendamment du masque, il y a un enduit noir qui est un anti-halo. Sans cela, quand les rayons lumineux atteignent le bord et le fond du film, un reflet vient se superposer à l'image et donc fait un léger halo. C'est à peine visible, mais le point n'est pas net. Le noir de l'anti-halo permet d'éviter cela en absorbant la lumière qui a traversé le film. Sur les pellicules noir et blanc donc, cet enduit n'existe pas. Mais le film est très légèrement gris, donc le halo est suffisamment diminué pour que ça ne marque pas. Ça c'est la théorie. Par contre, comme cette pellicule est un bout de plastique enroulé sur de la gélatine, à chaque début de prise, une petite friction survient et ce plastique se charge en électricité. Exactement comme quand vous enlevez un pull-over. Une étincelle se fait et marque la pellicule. On appelle cela des effluves, comme des petits dessins, blancs, très fins. Alors plus la pellicule est sensible, forcément plus ces effluves sont impressionnés. Dans Alphaville, avec la HPS, on avait sans arrêt ce genre de problèmes. Donc à chaque fois que nous avions une bonne prise, nous changions le magasin. Ce phénomène se produit dans certains cas, en fonction d'un degré hygrométrique qui n'est pas mesurable. Dans des endroits très chauds et très humides, par exemple, on sait qu'il n'y en aura pas. Mais ailleurs... De plus, comme les pellicules d'Alphaville étaient envoyées par avion à Londres pour le développement, elles étaient inévitablement secouées. Donc les effluves pouvaient se produire dans la caméra, durant le transport ou au laboratoire, quand les bobines étaient déroulées.

À part ces contraintes techniques ou chimiques, le tournage de ces scènes à la luminosité parfois très vive a-til posé des obstacles majeurs?

La scène d'hôtel était assez compliquée à réaliser. Eddy entre dans le hall, monte dans I'ascenseur, la caméra I'accompagne, puis il longe le couloir pour regagner sa chambre. II n'y a qu'un seul plan, exactement ce que JeanLuc adore. Nous y avons passé un temps fou : il fallait que deux ascenseurs démarrent au même moment. C'étaient deux vieilles «bécanes» et, en appuyant simultanément sur les deux boutons, il y en avait toujours un pour s'enclencher avant l'autre. Indépendamment du fait que le plan était difficile à coordonner, il fallait qu'un technicien fasse le point dans le hall, qu'un autre le reprenne dans l'ascenseur pour le panoramique, une fois dans le couloir qu'un autre encore s'en charge. Sans compter qu'il fallait ajuster la lumière entre le hall, très éclairé, le boulevard extérieur, les ascenseurs, donc installer quand même des lampes. Mais comme nous étions en noir et blanc, il n'y avait pas de problème de balance de couleurs, donc nous pouvions monter ou descendre les lampes en fonction de la quantité de lumière souhaitée. Quand on baisse la lumière, elle devient jaune: en couleurs, ce ne serait pas passé, mais en noir et blanc, ça ne posait pas de problème.

Le premier passage à la couleur avec Godard, dans Une femme est une femme, a-t-il été problématique? Pas véritablement. Jean-Luc à l'époque avait une idée: quand on tourne en noir et blanc, on est en format 1,33; quand on tourne en couleurs, on est en cinémascope. Donc Une femme est une femme a été tourné, forcément, en cinémascope. Jean-Luc est assez fasciné par la couleur : dans Pierrot le fou, il a «barbouillé » certains aspects, dans Le Mépris, également, c'est lui qui a peint les statues. Ça fait partie de son univers. 
Dans la scène d'ouverture du Mépris, justement, entre Bardot et Piccoli, les teintes du début, la lampe qui s'allume brusquement, tout était-il très précisément orchestré?

Juste après le générique? Ce n'est pas moi qui l'ai tournée. Vous savez que le film a été financé par les Américains; on dit «Rome Paris Films», mais ce sont eux qui ont donné six cents millions de l'époque pour sa réalisation. Et les Américains voulaient d'abord que l'on voit Bardot nue. Jean-Luc, ça ne l'intéressait pas. Il y a eu deux réactions: initialement le film devait être tiré en technicolor, mais plus question (le technicolor suppose beaucoup de copies sinon il n'est pas rentable), donc nous avons ramené le film en France, puis ils ont exigé une scène avec Bardot nue, d'où la scène du début. Mais c'était longtemps après, moi j'étais sur un autre tournage.

\section{Étiez-vous content que Le Mépris échappe finalement au technicolor ?}

J'aurais bien aimé qu'il soit tiré en technicolor, pour voir. En tout cas, je me souviens d'un problème ridicule. Le Mépris a été tourné en Italie et nous le faisions développer chez Technicolor à Rome. Dans le cadre d'un accord mondial, Kodak s'était réservé la fabrication de la pellicule négative, laissant celle de la positive, dans d'autres pays que la France, aux autres fabricants. En Italie, par exemple, la positive était faite par Ferrania. Nous avons tourné une dizaine de jours sans voir les «rushes», on se contentait de contacter le laboratoire pour connaître les lumières de tirage. Pour savoir techniquement ce qui se passe, si on connaît bien la façon dont on travaille, il suffit de prendre connaissance des rapports numériques. On sait ainsi si l'image est bonne, surexposée, sous-exposée, s'il y a un peu trop de jaune, de rouge, etc. Tout semblait correct, donc nous étions sereins. Finalement nous allons voir les « rushes». Complètement effondrés! Le film était tout jaunâtre, sépia, il n’y avait pas de couleurs vives, tout était triste. On l'explique au laboratoire. "C'est parce que vous êtes habitués à la Kodak, mais en réalité il n’y aucun problème», répondent-ils. Ils ont envoyé quelqu'un à Paris acheter de la pellicule Kodak, ont tiré deux scènes en technicolor pour montrer la différence. Là, le film était avec les couleurs telles que vous les connaissez. Donc on n'a plus regardé les «rushes» qu'en Ferrania, juste pour vérifier les cadrages, sans se soucier de la lumière. Sur ce, on ramène le film à Paris, on visionne la première copie faite sur pellicule Kodak. Et on a trouvé ça effrayant tellement c'était coloré. On s'était habitués pendant dix semaines à la Ferrania, on trouvait ça très bien!

Dans le long plan du Mépris, à la villa Malaparte, y avait-il un dispositif pour éviter le contre-jour créé par la grande baie vitrée?

Nous avions été obligés de tapisser toutes les vitres de gélatine. Mais il y avait en permanence des courants d'air, donc par moments des vibrations. Or il fallait absolument ne pas avoir le moindre reflet. Les plans longs sont toujours difficiles. Dans la villa Malaparte, le seul problème tenait aux positionnements de la caméra, car comme il faisait beau, on n'a pas été confrontés à une fausse teinte extérieure. Parce que dans la région parisienne, par exemple, au moindre nuage...

Avec d'autres cinéastes que Godard, comment évoquez-vous les partis pris de lumière, comment se passent la communication et la connivence? Y a-t-il des incompréhensions de langage?

Je vais vous dire: avec les metteurs en scène que je connais bien, avec lesquels je m'entends, nous avons plutôt tendance à parler de tout autre chose que du film en tournage. Mais c'est aussi constructif. Et puis, à partir d'un certain moment, on a compris ce qu'ils veulent, on se doute qu'on va pouvoir faire comme ça, et il est inutile d'en parler sinon pour en dire trois mots sans donner d'explication. Pour le reste, la question du langage est très compliquée. Je prends souvent l'exemple du cinéaste qui me demande, pour des photos de nuit, une nuit noire, qui veut en finir avec les effets habituels, mais qui n'envisage pas que la scène ne soit pas éclairée, qu'on ne voie pas la vedette. Inévitablement, on va éclairer, mettre un peu de bleu dedans et on retrouve le train-train quotidien. En somme, quand un metteur en scène demande du noir, il ne faut pas qu'on fasse du noir. 
Ce n'est probablement pas le dialogue que vous avez échangé avec Truffaut pour la première scène de Tirez sur le pianiste, où la nuit est bel et bien noire...

Sur Le Pianiste on a eu un certain nombre de problèmes, $d^{\prime}$ incidents techniques; on oublie souvent que ces filmslà ont été faits il y a un quart de siècle, c'est-à-dire à une époque où les tournages dans la rue ne se faisaient pas normalement, mais dans la cour des studios, avec des projecteurs qui n'éclairaient pas comme on voulait. Le long de la rue où cavale le frère d'Aznavour, nous n'avions pas d'autre solution que d'installer des lampes à réflecteur dans les arbres. Une trentaine. Mais ces lampes cassent dès qu'une goutte d'eau tombe dessus. Il s'est mis justement à pleuvoir et une dizaine d'entre elles se sont éteintes. Comme nous n'avions pas le temps de les changer, le comédien passe à certains moments dans la lumière et à d'autres dans le noir complet. Aujourd'hui, les lampes auraient été camouflées. Parfois, des contraintes techniques donnent ainsi un résultat qu'on peut juger finalement acceptable.

Y a-t-il des cinéastes particulièrement sensibles à la qualité de la lumière?

Oui, quelques-uns, mais à certains moments seulement. Je n’ai jamais vu un cinéaste attentif à la lumière sur la totalité d'un tournage.

S'agissant de votre recherche personnelle, quelle est votre relation à la lumière, dans votre travail comme dans votre observation quotidienne?

Je suis un fanatique des peintres hollandais, avec ces grandes traînées de lumière, ces grands pans d'ombre incluant des détails que l'on distingue à peine. Je suis fasciné par ce genre d'œuvres, Franz Hals, Rembrandt, toute une mouvance hollandaise. Cette idée de contraste de l'ombre et de la lumière me plaît beaucoup. Mais, quand on regarde la photographie que je fais, il n'y a absolument aucun rapport! Dans le cinéma, c'est en effet très compliqué à utiliser, sinon pour des polars ou des films de ce genre. C'est compliqué parce qu'on apporte une certaine violence dans l'image qui ne s'accorde par forcément avec le descriptif du tournage. Pourtant, les peintres hollandais, c'est une chose toute simple; on peut regarder pendant des heures, se demander ce qu'il pourrait y avoir dans cette ombre dans laquelle on ne voit rien, mais où il y a sûrement quelque chose à voir. Mais ce n'est pas une image de cinéma, parce qu'on ne pourrait pas la regarder aussi longtemps, parce qu'une autre image arriverait à la place.

Néanmoins le début de Vivre sa vie présente ces contrastes, sur le visage en gros plan d'Anna Karina, éclairé de face et de profil, une arête lumineuse sur le côté et une tache de lumière au niveau de la nuque...

Oui, quand je peux le faire je le fais, mais ce n'est guère envisageable sur la totalité d'un film. Je critique les metteurs en scène, mais quand j'ai réalisé Hoa Binh, je l'ai fait de façon relativement classique. De nombreux plans étant tournés dans la rue, je ne pouvais pas brusquement travailler la lumière des scènes d'intérieur jusqu'à créer une rupture, qui aurait en même temps miné la crédibilité du récit.

Sauf quand le récit porte justement sur ces peintres, dans Passion par exemple.

Le seul tableau filmé ressemblant exactement à l'original, c'était la Ronde de nuit ${ }^{1}$. Je me souviens que, pour L'Entrée des croisés à Constantinople², Jean-Luc souhaitait que la scène soit ressemblante mais pas tout à fait, ce qui avait posé quelques difficultés aux décorateurs. Cela a été long à mettre en place, nous avions utilisé tous les projecteurs disponibles dans le studio et mis trois générateurs en route.

Lorsque vous avez travaillé personnellement la lumière, quelle était la fonction que vous lui avez accordée? Était-elle pour vous surtout un opérateur de visibilité, servait-elle aussi à créer une ambiance?

J'essaie toujours de faire en sorte que la lumière soit en accord avec l'émotion qu'on essaie de créer, des émotions 
comme la peur, par exemple, un sentiment que le metteur en scène veut installer. C'est à ce niveau-là que j'essaie de travailler.

$\mathrm{Y}$ aurait-il alors une sorte de grammaire, une correspondance entre une émotion et une forme de lumière, diffuse, directionnelle, colorée ou autre? Est-ce que vous avez l'impression qu'il y a des grammaires plus ou moins fixes?

Non, je n'ai pas ce sentiment. La grammaire, je la conçois davantage pour la mise en scène. Griffith disait à un moment donné « Nous avons découvert le langage universel et, juste à ce moment-là, le cinéma parlant est entré en fonction », ce qui a rendu définitivement caduque la thèse $d^{\prime}$ un langage universel. Pour moi, la grammaire, ce sont des histoires contredites mais qui fonctionnent quand même.

Avez-vous l'impression que le travail de la lumière a développé votre sensibilité à la lumière quotidienne?

Oui. Il y a des endroits où la lumière est différente. Nous parlions du Mépris, on me demande fréquemment comment j'ai obtenu ces couleurs, mais ce sont les couleurs de Capri et de la Méditerranée. En Italie, dès qu'on décide d'aller un peu au sud de Rome, la lumière est particulière. Curieusement il y a une autre région où la lumière est différente, c'est autour du lac Léman, en Suisse. Près de Rolle, où vit Godard. La lumière est vraiment très belle, là.

Une dernière question. Dans le fameux plan du générique du Mépris, où vous apparaissez comme chefopérateur, quand vous regardez au travers d'un œilleton avant le panoramique, est-ce un geste nécessaire?

Non, Jean-Luc me l'avait demandé mais jamais on ne ferait ça! II s'agit d'un verre de contraste. Certains sont pour la couleur, panchromatiques, d'autres pour le noir et blanc. Quand on éclaire une scène, cela donne une idée de la valeur des contrastes. Souvent, sur les caméras, la Mitchell par exemple, il est intégré au viseur: il suffit $d^{\prime}$ appuyer sur un bouton. Cela permet de voir si un endroit n'est pas un peu trop éclairé, ou pas assez: on a les valeurs des hautes et basses lumières. Et comme ce verre est foncé, un peu plus foncé qu'une paire de lunettes de soleil, quand il y a des nuages, on regarde au travers pour vérifier qu'un nuage ne va pas passer devant le soleil. Mais là, manifestement, le ciel était très bleu, je n'avais aucune raison de l'utiliser.

Paris, 4 décembre 2002

1. Rembrandt, Ronde de nuit (ou la Compagnie du Capitaine Frans Banning Cocq). Huile sur toile, 1642, Rijkmuseum, Amsterdam. 2. Eugène Delacroix, L'Entrée des croisés à Constantinople. Huile sur toile, 1840. Musée du Louvre, Paris.

Filmographie sélective

Raoul Coutard, chef-opérateur :

1957 La Passe du diable,

Pierre Schoendoeffer et Jacques Dupont.

1958 Pêcheur d'Islande, Pierre Schoendoeffer.

1959 À bout de souffle, Jean-Luc Godard.

1960 Tirez sur le pianiste, François Truffaut. Le Petit Soldat, Jean-Luc Godard. Lola, Jacques Demy.

1961 Une femme est une femme, Jean-Luc Godard. Jules et Jim, François Truffaut.

1962 Vivre sa vie, Jean-Luc Godard. Vacances portugaises, Pierre Kast.

1963 Les Carabiniers, Jean-Luc Godard. Le Mépris, Jean-Luc Godard.

1964 Bande à part, Jean-Luc Godard. La Peau douce, François Truffaut. La 317 e Section, Pierre Schoendoerffer. Une femme mariée, Jean-Luc Godard.

1965 Alphaville, Jean-Luc Godard. Pierrot le fou, Jean-Luc Godard.

1966 Le Marin de Gibraltar, Tony Richardson. Made in USA, Jean-Luc Godard. Deux ou trois choses que je sais d'elle, Jean-Luc Godard.

1967 L'Espion, Raoul Levy. La Chinoise, Jean-Luc Godard. La mariée était en noir, François Truffaut. $Z$, Costa Gavras.

1970 L'Aveu, Costa Gavras.

La Liberté en groupe, Edouard Molinaro.

1977 Le Crabe-tambour, Pierre Schoendoerffer.

1981 Passion, Jean-Luc Godard.

1983 Prénom Carmen, Jean-Luc Godard. La Diagonale du fou, Richard Dembo.

1985 Max mon amour, Nagisa Oshima.

1988 Peaux de vaches, Patricia Mazuy.

1989 Il gèle même en enfer, Jean-Pierre Mocky.

1992 La Naissance de I'amour, Philippe Garrel.

Raoul Coutard, réalisateur :

1969 Hoa Binh.

1970 Jolly Green.

1975 La Ville étranglée (reportage en coll. avec Jean Larteguy).

1979 La Légion saute sur Kolwezi.

1982 SAS à San Salvador. 